\title{
The Critical Catalog: Giving Voice to Diverse Library Materials through Provocative Design
}

\author{
Rachel Ivy Clarke \\ Syracuse University \\ rclark01@syr.edu
}

\author{
Sayward Schoonmaker \\ Syracuse University \\ sschoonm@syr.edu
}

\begin{abstract}
Although laudable strides have been made to highlight and provide access to diverse library materials about and made by traditionally marginalized communities, current approaches are curatorial, non-scalable, and non-systematic. Using a critical design approach, we address how libraries might move beyond curatorial practices with the proposal of a "Critical Catalog" that advocates for diverse materials and discusses the problems and challenges of categorizing identity. The proposed provocative catalog offers the possibility to raise awareness of diverse library materials; expose readers to new and different resources, ideas and cultures; alter reading habits; and ultimately provide more equitable representation by preventing the inadvertent and unintentional erasure of diverse library materials, thus giving a stronger voice to marginalized communities.
\end{abstract}

\section{Keywords}

Diversity, Metadata, Knowledge Organization

\section{INTRODUCTION AND PROBLEM STATEMENT}

Diversity is a core value of American librarianship (American Library Association 2004) with a specific call for librarians to provide access to library resources for diverse communities and from diverse populations (American Library Association 2013). People from traditionally marginalized communities in the United States, including women and people of non-traditional genders, people of color, indigenous peoples, people identifying as LGBTQIA+, and people with disabilities, need access to books and library resources about or created by people like themselves so that they can see their identities, stories, and experiences reflected in contemporary media, and feel empowered to create new works. People from traditionally mainstream communities also benefit from exposure to and consumption of media about and by diverse peoples, to see the world from alternative perspectives and learn empathy. As bastions of reading and literacy, American libraries are uniquely positioned to support these benefits by providing access to and promoting diverse books and other library resources.

81" Annual Meeting of the Association for Information Science \& Technology I Vancouver, Canada I Nov. 10 - 14, 2018

Author(s) Retain Copyright
In recent years, libraries have drawn on various techniques to achieve this goal. Although the number of published resources in the U.S. by and about diverse peoples is disproportionally small (Cooperative Children's Book Center 2017), strategies to provide access to and promotion of these resources are emerging. Most attempts take the form of booklists or bibliographies, or promotional events and programming. Library workers also draw on traditional library services such as book-talking, collection development, readers' advisory, and displays or exhibits of physical materials in the library (Killeen 2015). All of these approaches should be lauded as an important step in helping connect readers with diverse materials. However, these approaches face limitations. The majority of these examples rely on an individual person or organization to curate a list or collection of resources, or offer recommendations tailored to an individual reader. This approach is often ad-hoc, unsystematic, and not scalable. Additionally, most of these approaches require readers to have previously established interest in seeking diverse materials. Such self-selection can create a kind of "filter bubble" where people who might benefit from reading diverse resources do not realize those resources exist, thus reifying the erasure of minority and marginalized groups. How then, can libraries move beyond the artisanal, curation-based approaches to promoting diverse resources in order to encourage a wider readership of both diverse and mainstream audiences?

\section{A DESIGN PROPOSAL: THE CRITICAL CATALOG}

To achieve this goal, we propose a provocative approach: the creation of a new library catalog that advocates for diversity and exposes library users and readers to resources from populations traditionally marginalized in literature and publishing by designing a system where the default is no longer the white heteronormative male author. Picture this: a user searches the keyword "childhood" and the Critical Catalog returns books created by women and people of non-traditional genders, people of color, indigenous peoples, people identifying as LGBTQIA+, and people with disabilities - a kind of "affirmative action" catalog. Instead of user-applied filtering or other traditional attributes, this catalog challenges the library status quo by returning only results from marginalized authors (See Figure 1). 
The Critical Catalog

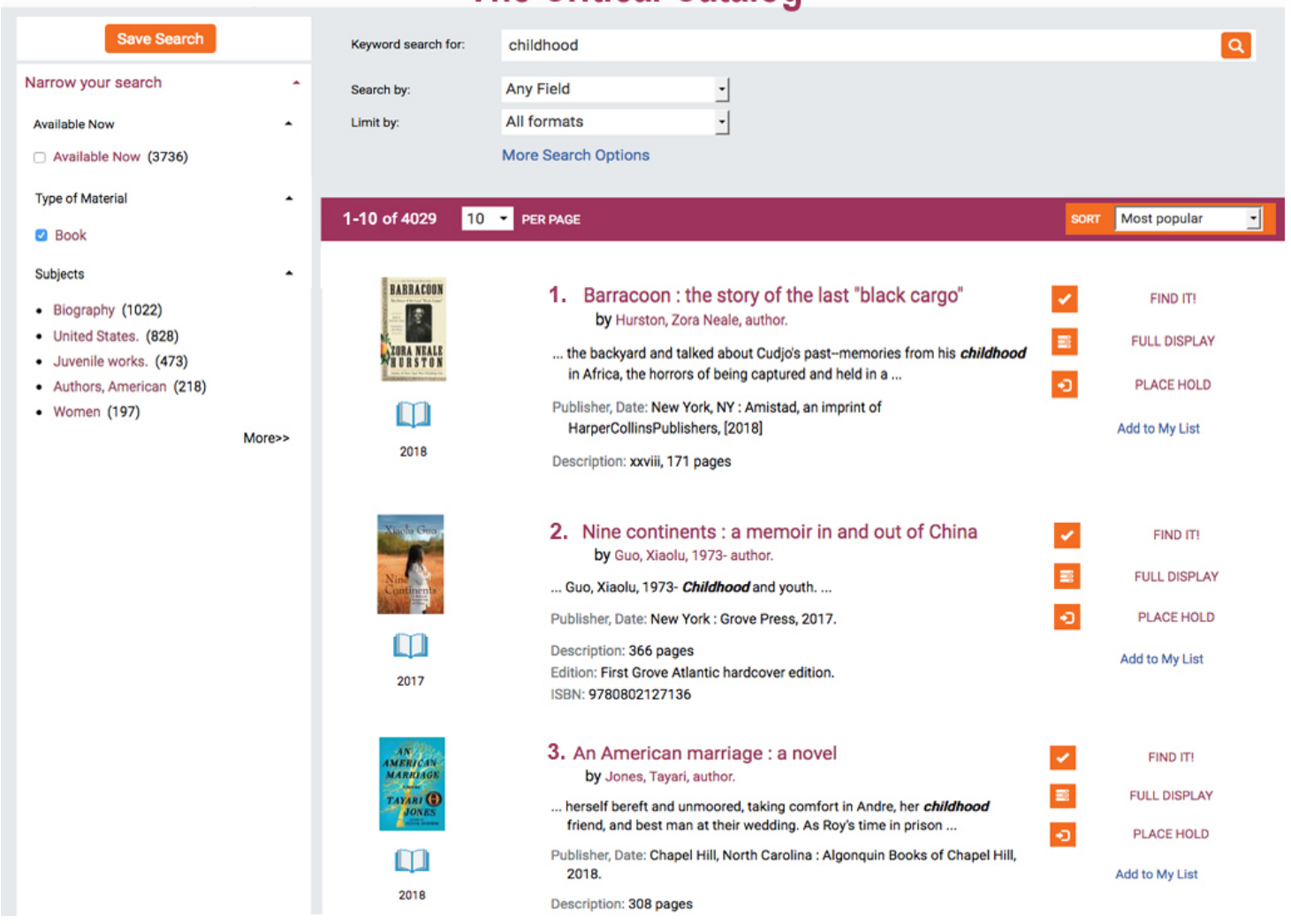

Figure 1: Mock-up image of potential Critical Catalog

\section{APPROACH: METADATA FOR DIVERSITY}

To enable such a project, metadata reflecting diversity is necessary - something traditional library catalog records do not include. Although the number of data elements included in bibliographic description has increased over time, access points - data elements that offer a point of entry to a collection and a means to collocate similar materials (Reitz 2014) - have changed little since Cutter's articulation of the main objectives of library catalogs (Clarke 2015). Additionally, information describing creators of library resources is typically not included in bibliographic records but instead in authority records, which serve to maintain consistency of verbal forms of creator's names and document relationships between forms of names (Taylor and Joudry 2009, 249). Fully describing a creator's physical, intellectual, or other characteristics -including their status as regards diversity - was never the intention nor the purview of library authority data. Therefore, data regarding gender, ethnicity, sexuality, or other characteristics that might offer more in-depth information about a creator, is traditionally only recorded if it serves to disambiguate one creator from another.

Recent developments in and evolutions of library metadata have influenced changes to this traditional approach. The semantic web is one major development that aims to "bring structure to the meaningful content of web pages, creating an environment where software agents roaming from page to page can readily carry out sophisticated tasks for users," such as more precise and automated searching (Berners-
Lee, Hendler and Lassila 2001). Semantic Web technologies also offer more openly accessible and interoperable metadata, allowing data from non-library systems to be integrated into library systems, and vice versa (Bermes 2013 , 118). Another major shift is the evolution of library cataloging rules and guidelines, such as the development of Resource Description and Access (RDA), which includes more affordances for recording descriptions that support these new data-sharing models. For instance, unlike previous cataloging rules, RDA allows for the recording of data such as profession/field of work and gender when describing a person (Dobreski and Kwasnik 2017). But these recent additions are not without issues. Billey, Drabinski and Roberto (2014) criticize the inclusion of gender metadata in authority records, as the suggested controlled vocabularies limit the ability to describe creators outside the binary norm and gender fluidity over time. Additionally, they argue that the trouble caused by recording descriptors for gender and other elements like ethnicity outweighs any retrieval or disambiguation functions, claiming that use of gender for a retrieval access point is not an objective of library cataloging (420). Our own ongoing field scan of metadata describing diversity shows that particular institutions use demographic metadata such as gender, sexuality, race, ethnicity, religion, and physical/mental differences to highlight diverse materials rather than disambiguate creators' identities. Each institution employs different levels of granularity in describing diversity of creators and of resources. For example, the Queer Cartoonist Database offers 18 values for 
gender while NoveList's gender values are limited to "All," "Male," and "Female." Conversely, GoodReads employs user-generated tagging to describe resources. GoodReads' gender-specific term tags vary and are housed with all tags under the element "genre." These examples raise problematic and important questions about who makes these values/elements and how this naming affects the creator's agency or a resource's categorization. Identifying authors or resources by gender risks outing an author whose gender carries the possibility of discrimination or violence (Thompson 2016) and labeling a resource by descriptors that carry no relevance to its aboutness (Billey, Drabinski, and Roberto 2014).

Given the problematic aspects of delineating diverse characteristics of creators/resources, which may inappropriately label already marginalized populations, we propose a thought experiment: that labeling characteristics traditionally used to identify diverse creators/resources may be a problematic solution. Instead, we propose the Critical Catalog functions on a flipped script: instead of labeling "diverse" materials, we propose to label materials created by or about cisgendered white able-bodied heteronormative men. Including demographic descriptors for this male category resists white normativity that frames this male category as "a neutral or standard category of human beings" (Morris 2016). This "norm" affords such identities freedom from stereotyping and diminished complexity. Hence, labeling the cis white hetero able-bodied male category potentially relinquishes marginalized groups from strident or incorrect categorization while de-stabilizing the patriarchal norm. Hence, flipping the narrative and making cis the "other" brings to light the power implications of classifying and categorizing information.

\section{CONCLUSION}

Most library catalogs are designed to meet traditional objectives: to find materials, to identify an entity, to select an entity, and to obtain access to materials (IFLA 1998). Yet alternative purposes and objectives exist, including navigation and discovery; education; social connection and interaction; and expression (Clarke 2014). Library information systems, such as classification schemes, thesauri, and digital collections, express persuasive rhetorical arguments that reflect various points of view (Feinberg 2010). These information systems can also erase or bury particular perspectives (e.g., Bowker and Star 1999; Adler 2017). Because these systems express arguments regardless of their intention to do so, the purpose of expression is implicit in every system-and therefore every catalog-ever created. Therefore, it is not a question of whether or not expression should be a purpose of library catalogs, but rather a question of what should be expressed. With diversity as a core value of American librarianship, libraries are charged with promoting diverse materials and advocating for diverse populations. Therefore, they need to explicitly express such aims in their catalogs in ways that advocate for diverse materials, encourage exposure of such materials to a broader audi- ence, and present the unintentional erasure of such materials in library collections. Even as a mere thought experiment, our proposed provocative system offers the possibility to raise awareness of diverse library materials; expose readers to new and different resources, ideas and cultures; alter reading habits; and ultimately provide more equitable representation by preventing the inadvertent and unintentional erasure of diverse library materials, giving voice to marginalized communities.

\section{ACKNOWLEDGEMENTS}

We wish to acknowledge and express our gratitude for funding support from the 2018 OCLC/ALISE Library \& Information Science Research Grant Program (LISRGP).

\section{REFERENCES}

Adler, M. (2017). Cruising the Library: Perversities in the Organization of Knowledge. New York: Fordham University Press.

American Library Association (2004). Core Values of Librarianship.

http://www.ala.org/advocacy/intfreedom/statements pols/corevalues

American Library Association (2013). ALA Policy Manual Section B: Positions and Public Policy Statements. http://www.ala.org/aboutala/sites/ala.org.aboutala/files/co ntent/governance/policymanual/cd_10_2_Section\%20B\% 20New\%20Policy\%20Manual-1\%20\%28final\%206-132017\%20with\%20TOC\%29_0.pdf Section B.3.

Bermes, E. (2013). Enabling Your Catalogue for the Semantic Web. In Chambers, S. (ed.) Catalogue 2.0: The Future of the Library Catalogue, Chicago: NealSchulman.

Berners-Lee, T., Hendler, J. and Lassila, O. (2001). The Semantic Web. Scientific American 284(5): 34-43.

Billey, A., Drabinski, E. and Roberto, K. R. (2014). What's gender Got to Do With It? A Critique of RDA 9.7. Cataloging \& Classification Quarterly 52(4): 412-421.

Bowker, G. C. and Star, S. L. (1999). Sorting Things Out: Classification and its Consequences. Cambridge, Mass: MIT Press.

Clarke, R.I. (2014). Find, Identify, Select...Socialize?: Alternative Objectives of Library Catalogs. In ASIS\&T 2014: Proceedings of the 77th ASIS\&T Annual Meeting, Seattle, Washington.

Clarke, R. I. (2015) Breaking Records: The History of Bibliographic Records and Their Influence in Conceptualizing Bibliographic Data. Cataloging \& Classification Quarterly 53(3-4): 286-302.

Cooperative Children's Book Center (2017). Publishing Statistics on Children's Books about People of Color and First/Native Nations and by People of Color and 
First/Native Nations Authors and Illustrators. http://ccbc.education.wisc.edu/books/pcstats.asp

Dobreski, B. and Kwasnik. B. (2017). Changing depictions of persons in library practice: spirits, pseudonyms and human books. In Knowledge Organization-What's the Story? Proceedings of the 2017 ISKO UK Biennial Conference.

Feinberg, M. (2010). Two kinds of evidence: how information systems form rhetorical arguments. Journal of Documentation 66(4): 491-512.

IFLA Study Group on the Functional Requirements for Bibliographic Records. (1998). Functional Requirements for Bibliographic Records: Final Report. Munich: K.G. Saur Verlag.
Killeen, E. B. (2015). "\#WeNeedDiverseBooks!" Teacher Librarian 42(5): 52-63.

Morris, M. (2016). Standard white: Dismantling white normativity. California Law Review 104(4), 949.

Reitz, J. M. (2014). "access points." Online Dictionary of Library and Information Science. Santa Barbara, CA: ABC-CLIO. clio.com/ODLIS/odlis_a.aspx\#accesspoint http://www.abc-

Taylor, A.G., and Joudrey, D.N. (2009). The Organization of Information, 3rd ed. Westport, Conn: Libraries Unlimited.

Thompson, K. J. (2016). More than a name: A content analysis of name authority records for authors who selfidentify as trans. Library Resources \& Technical Services, 60(3), 140. doi:10.5860/1rts.60n3.140 